\title{
A new class of generalized polynomials involving Laguerre and Euler polynomials
}

\author{
Nabiullah $\operatorname{Khan}^{1}$ (D), Talha Usman ${ }^{2}$ (D), Junesang Choi*3 (D) \\ ${ }^{1}$ Department of Applied Mathematics, Faculty of Engineering and Technology, Aligarh Muslim \\ University, Aligarh 202002, India \\ ${ }^{2}$ Department of Mathematics, School of Basic and Applied Sciences, Lingaya's Vidyapeeth, Faridabad \\ 121002, Haryana, India \\ ${ }^{3}$ Department of Mathematics, Dongguk University, Gyeongju 38066, Republic of Korea
}

\begin{abstract}
Motivated by their importance and potential for applications in a variety of research fields, recently, numerous polynomials and their extensions have been introduced and investigated. In this paper, we modify the known generating functions of polynomials, due to both Milne-Thomsons and Dere-Simsek, to introduce a new class of polynomials and present some involved properties. As obvious special cases of the newly introduced polynomials, we also introduce power sum-Laguerre-Hermite polynomials and generalized Laguerre and Euler polynomials and give certain involved identities and formulas. We point out that our main results, being very general, are specialised to yield a number of known and new identities involving relatively simple and familiar polynomials.
\end{abstract}

Mathematics Subject Classification (2020). 05A10, 05A15, 11B68

Keywords. Milne-Thomsons polynomials, Dere-Simsek polynomials, Laguerre polynomials, Hermite polynomials, Euler polynomials, generalized Laguerre-Euler polynomials, summation formulae, symmetric identities

\section{Introduction and preliminaries}

The two variable Laguerre polynomials $L_{n}(x, y)$ are generated by (see $[8,18]$ )

$$
\frac{1}{1-y t} \exp \left(\frac{-x t}{1-y t}\right)=\sum_{n=0}^{\infty} L_{n}(x, y) t^{n} \quad(|y t|<1) .
$$

Also, equivalently, the polynomials $L_{n}(x, y)$ are given by (see $\left.[9,18]\right)$

$$
\mathrm{e}^{y t} C_{0}(x t)=\sum_{n=0}^{\infty} L_{n}(x, y) \frac{t^{n}}{n !}
$$

\footnotetext{
*Corresponding Author.

Email addresses: nukhanmath@gmail.com (N.U. Khan), talhausman.maths@gmail.com (T. Usman), junesang@dongguk.ac.kr (J. Choi)

Received: 19.04.2019; Accepted: 05.04.2020
} 
where $C_{0}(x)$ denotes the 0th order Tricomi function. The $n$th order Tricomi functions $C_{n}(x)$ are generated by

$$
\exp \left(t-\frac{x}{t}\right)=\sum_{n=0}^{\infty} C_{n}(x) t^{n} \quad(t \in \mathbb{C} \backslash\{0\}, x \in \mathbb{C}) .
$$

We have

$$
C_{n}(x)=\sum_{r=0}^{\infty} \frac{(-1)^{r} x^{r}}{r !(n+r) !} \quad\left(n \in \mathbb{N}_{0}\right) .
$$

The Tricomi functions $C_{n}(x)$ are connected with the Bessel function of the first kind $J_{n}(x)$ (see [7]):

$$
C_{n}(x)=x^{-\frac{n}{2}} J_{n}(2 \sqrt{x}) .
$$

Here and throughout, we denote $\mathbb{C}, \mathbb{R}, \mathbb{R}^{+}, \mathbb{Z}$, and $\mathbb{N}$ by the sets of complex numbers, real numbers, positive real numbers, integers, and positive integers, respectively, and let $\mathbb{N}_{0}:=\mathbb{N} \cup\{0\}$.

From (1.2) and (1.4), we find

$$
L_{n}(x, y)=n ! \sum_{s=0}^{n} \frac{(-1)^{s} x^{s} y^{n-s}}{(s !)^{2}(n-s) !}=y^{n} L_{n}(x / y),
$$

where $L_{n}(x)$ are the ordinary Laguerre polynomials (see, e.g., $[1,26]$ ). We thus have

$$
L_{n}(x, 0)=\frac{(-1)^{n} x^{n}}{n !}, \quad L_{n}(0, y)=y^{n}, \quad L_{n}(x, 1)=L_{n}(x) .
$$

Milne-Thomson [22] defined polynomials $\Phi_{n}^{(\alpha)}(x)$ of degree $n$ and order $\alpha$ by the following generating function

$$
f(t, \alpha) \mathrm{e}^{x t+g(t)}=\sum_{n=0}^{\infty} \Phi_{n}^{(\alpha)}(x) \frac{t^{n}}{n !},
$$

where $f(t, \alpha)$ is a function of $t$ and $\alpha \in \mathbb{Z}$ and $g(t)$ is a function of $t$. Then, by choosing some explicit functions of $f(t, \alpha)$ and $g(t)$, Milne-Thomsons [22] presented several interesting properties for polynomials such as Bernoulli polynomials and Hermite polynomials.

Derre and Simsek [10] made a slight modification of the Milne-Thomson's polynomials $\Phi_{n}^{(\alpha)}(x)$ to give polynomials $\Phi_{n}^{(\alpha)}(x, \nu)$ of degree $n$ and order $\alpha$ by means of the following generating function

$$
G(t, x ; \alpha, \nu):=f(t, \alpha) \mathrm{e}^{x t+h(t, \nu)}=\sum_{n=0}^{\infty} \Phi_{n}^{(\alpha)}(x, \nu) \frac{t^{n}}{n !},
$$

where $f(t, \alpha)$ and $h(t, \nu)$ are functions of $t$ and $\alpha \in \mathbb{Z}$ and $t$ and $\nu \in \mathbb{N}_{0}$, respectively, which are analytic in a neighborhood of $t=0$. Observe that $\Phi_{n}^{(\alpha)}(x, 0)=\Phi_{n}^{(\alpha)}(x)$ (see, for details, [22]).

By setting $f(t, \alpha)=\left(\frac{t}{e^{t}-1}\right)^{\alpha}$ in $(1.9)$, in [18], we introduced the polynomials $B_{n}^{(\alpha)}(x, \nu)$ defined by

$$
\left(\frac{t}{\mathrm{e}^{t}-1}\right)^{\alpha} \mathrm{e}^{x t+h(t, \nu)}=\sum_{n=0}^{\infty} B_{n}^{(\alpha)}(x, \nu) \frac{t^{n}}{n !} .
$$

Here, by choosing $f(t, \alpha)=\left(\frac{2}{e^{t}+1}\right)^{\alpha}$ in (1.9), we introduce the following polynomials $E_{n}^{(\alpha)}(x, \nu)$ defined by

$$
\left(\frac{2}{\mathrm{e}^{t}+1}\right)^{\alpha} \mathrm{e}^{x t+h(t, \nu)}:=\sum_{n=0}^{\infty} E_{n}^{(\alpha)}(x, \nu) \frac{t^{n}}{n !}
$$


We find that the polynomials $E_{n}^{(\alpha)}(x, \nu)$ are related to not only Euler polynomials but also the Hermite polynomials. For example, if $h(t, 0)=0$ in $(1.11)$, we have

$$
E_{n}^{(\alpha)}(x, 0)=E_{n}^{(\alpha)}(x)
$$

where $E_{n}^{(\alpha)}(x)$ denote the Euler polynomials of higher order defined by means of the following generating function (see, e.g., [27, p. 88])

$$
F_{E}(t, x ; \alpha):=\left(\frac{2}{\mathrm{e}^{t}+1}\right)^{\alpha} \mathrm{e}^{x t}=\sum_{n=0}^{\infty} E_{n}^{(\alpha)}(x) \frac{t^{n}}{n !} .
$$

We find

$$
F_{E}(t, 0 ; \alpha):=F_{E}(t ; \alpha)=\left(\frac{2}{\mathrm{e}^{t}+1}\right)^{\alpha}=\sum_{n=0}^{\infty} E_{n}^{(\alpha)} \frac{t^{n}}{n !},
$$

where $E_{n}^{(\alpha)}$ are generalized Euler numbers. For more information about Euler numbers and Euler polynomials, we refer the reader, for example, to [3, 20,21,27].

Taking $h(t, y)=y t^{2}$ in (1.11), we get the generalized Hermite-Euler polynomials of two variables ${ }_{H} E_{n}^{(\alpha)}(x, y)$ introduced by Pathan [23]:

$$
\left(\frac{2}{\mathrm{e}^{t}+1}\right)^{\alpha} \mathrm{e}^{x t+y t^{2}}=\sum_{n=0}^{\infty}{ }_{H} E_{n}^{(\alpha)}(x, y) \frac{t^{n}}{n !} .
$$

Note that the polynomials ${ }_{H} E_{n}^{(\alpha)}(x, y)$ generalize Euler numbers, Euler polynomials, Hermite polynomials, and Hermite-Euler polynomials ${ }_{H} E_{n}(x, y)$ introduced by Dattoli et al. [6, p. 386, Eq. (1.6)]:

$$
\frac{2}{\mathrm{e}^{t}+1} \mathrm{e}^{x t+y t^{2}}=\sum_{n=0}^{\infty} H E_{n}(x, y) \frac{t^{n}}{n !} .
$$

The sum of integer power (simply, power sum)

$$
S_{k}(\mathrm{n}):=\sum_{j=0}^{\mathrm{n}} j^{k} \quad\left(k \in \mathbb{N}_{0} ; \mathrm{n} \in \mathbb{N}\right)
$$

is generated by

$$
\sum_{k=0}^{\infty} S_{k}(\mathrm{n}) \frac{t^{k}}{k !}=1+\mathrm{e}^{t}+\mathrm{e}^{2 t}+\cdots+\mathrm{e}^{\mathrm{n} t}=\frac{\mathrm{e}^{(\mathrm{n}+1) t}-1}{\mathrm{e}^{t}-1} .
$$

Luo et al. [20,21] introduced the generalized Euler numbers $E_{n}(a, b)$ generated by

$$
\begin{gathered}
\Phi(t ; a, b)=\frac{2}{a^{t}+b^{t}}=\sum_{n=0}^{\infty} E_{n}(a, b) \frac{t^{n}}{n !} \\
\left(|t|<2 \pi ; n \in \mathbb{N}_{0} ; a, b \in \mathbb{R}^{+} \text {with } a \neq b\right) .
\end{gathered}
$$

Also, Luo et al. [20] introduced the generalized Euler polynomials $E_{n}(x ; a, b, \mathrm{e})$ generated by

$$
\begin{gathered}
\Phi(x, t ; a, b, \mathrm{e})=\frac{2 \mathrm{e}^{x t}}{a^{t}+b^{t}}=\sum_{n=0}^{\infty} E_{n}(x ; a, b, \mathrm{e}) \frac{t^{n}}{n !} \\
\left(|t|<2 \pi ; n \in \mathbb{N}_{0} ; a, b \in \mathbb{R}^{+} \text {with } a \neq b\right) .
\end{gathered}
$$

The 2-variable Hermite-Kampé de Fériet polynomials $H_{n}(x, y)$ (see $\left.[2,6]\right)$ are generated by

$$
\mathrm{e}^{x t+y t^{2}}=\sum_{n=0}^{\infty} H_{n}(x, y) \frac{t^{n}}{n !}
$$


Note that

$$
H_{n}(x, y)=n ! \sum_{r=0}^{\left[\frac{n}{2}\right]} \frac{y^{r} x^{n-2 r}}{r !(n-2 r) !}
$$

and $H_{n}(2 x,-1)=H_{n}(x)$ are the ordinary Hermite polynomials (see, e.g., [2]; see also [26, Chapter 11]). Dere and Simsek [10] generalized the polynomials $H_{n}(x, y)$ in (1.19) to define two variable Hermite polynomials $H_{n}^{(\ell)}(x, y)$ by the following generating function

$$
\mathrm{e}^{x t+y t^{\ell}}=\sum_{n=0}^{\infty} H_{n}^{(\ell)}(x, y) \frac{t^{n}}{n !} \quad(\ell \in \mathbb{N} \backslash\{1\}) .
$$

Very recently, Khan et al. [18, Eq. (20)] have introduced and investigated the following generalized Laguerre-Bernoulli polynomials

$$
\begin{aligned}
& \left(\frac{t}{a^{t}-b^{t}}\right)^{\alpha} \mathrm{e}^{y t+z t^{2}} C_{0}(x t)=\sum_{n=0}^{\infty}{ }_{L} B_{n}^{(\alpha)}(x, y, z ; a, b, \mathrm{e}) \frac{t^{n}}{n !} \\
& \left(\alpha, x, y, z \in \mathbb{C}, a, b \in \mathbb{R}^{+}, a \neq b,|t|<\frac{2 \pi}{|\ln a-\ln b|}\right) .
\end{aligned}
$$

Motivated by their importance and potential for applications in certain problems in number theory, combinatorics, classical and numerical analysis and other fields of applied mathematics, a number of certain numbers and polynomials, and their generalizations have recently been extensively investigated (see, e.g., [1-30]). Here, we also make a slight modification of Milne-Thomson polynomials $\Phi_{n}^{(\alpha)}(x)$ in (1.8) and Derre and Simsek polynomials $\Phi_{n}^{(\alpha)}(x, \nu)$ in (1.9) to define polynomials $\Phi_{n, \ell}^{(\alpha)}(x, y, \nu)$ by the following generating function

$$
\begin{aligned}
H(t, x, y ; \alpha, \nu):= & f(t, \alpha) \mathrm{e}^{x t+y t^{\ell}+h(t, \nu)}=\sum_{n=0}^{\infty} \Phi_{n}^{(\alpha, \ell)}(x, y, \nu) \frac{t^{n}}{n !} \\
& (x, y \in \mathbb{C} ; \ell \in \mathbb{N} \backslash\{1\}),
\end{aligned}
$$

where $f(t, \alpha)$ and $h(t, \nu)$ are functions of $t$ and $\alpha \in \mathbb{Z}$ and $t$ and $\nu \in \mathbb{N}_{0}$, respectively, which are analytic in a neighborhood of $t=0$. Obviously $\Phi_{n}^{(\alpha, \ell)}(x, 0, \nu)=\Phi_{n}^{(\alpha)}(x, \nu)$. Then we establish various identities involving the polynomials $\Phi_{n}^{(\alpha, \ell)}(x, y, \nu)$. Also, as special cases of the generalized generating function in (1.23), we introduce two new polynomials: power sum-Laguerre-Hermite polynomials and generalized Laguerre-Euler polynomials and investigate some involved properties.

Some of the results presented here will include certain known identities and formulas involving relatively simple and familiar numbers and polynomials as particular cases, which are easy for the interested reader to check (see, e.g., [8,12-17,21, 23, 24, 29, 30]).

Remark 1.1. The substitution

$$
f(t, \alpha)=\left(\frac{t}{a^{t}-b^{t}}\right)^{\alpha} C_{0}(x t), \quad h(t, \nu)=0, \quad \text { and } \quad \ell=2
$$

in (1.23) yields (1.22). So it may imply that the polynomials in (1.23) are more general than those in (1.22). The process and methods used in this paper follow from those employed in such works as [5,13,15-17] including, in particular, the very recent work [18].

\section{Some formulas involving the polynomials $\Phi_{n, \ell}^{(\alpha)}(x, y, \nu)$}

Here, we present certain formulas associated with the polynomials $\Phi_{n, \ell}^{(\alpha)}(x, y, \nu)$. To do this, we recall some formal manipulations of double series in the following lemma (see, e.g., [4], [17], [26, pp. 56-57], and [28, p. 52]). 
Lemma 2.1. The following identities hold:

$$
\begin{aligned}
& \sum_{n=0}^{\infty} \sum_{k=0}^{\infty} A_{k, n}=\sum_{n=0}^{\infty} \sum_{k=0}^{[n / p]} A_{k, n-p k} \quad(p \in \mathbb{N}) ; \\
& \sum_{n=0}^{\infty} \sum_{k=0}^{[n / p]} A_{k, n}=\sum_{n=0}^{\infty} \sum_{k=0}^{\infty} A_{k, n+p k} \quad(p \in \mathbb{N}) ; \\
& \sum_{N=0}^{\infty} f(N) \frac{(x+y)^{N}}{N !}=\sum_{n, m=0}^{\infty} f(m+n) \frac{x^{n}}{n !} \frac{y^{m}}{m !} .
\end{aligned}
$$

Here, the $A_{k, n}$ and $f(N)\left(k, n, N \in \mathbb{N}_{0}\right)$ are real or complex valued functions indexed by the $k, n$ and $N$, respectively, and $x$ and $y$ are real or complex numbers. Also, for possible rearrangements of the involved double series, all the associated series should be absolutely convergent.

Theorem 2.2. Let $\alpha \in \mathbb{Z}, \nu \in \mathbb{N}_{0}$, and $\ell \in \mathbb{N} \backslash\{1\}$. Then

$$
\begin{aligned}
& \Phi_{n}^{(\alpha, \ell)}\left(x_{1}+x_{2}, y, \nu\right)=\sum_{k=0}^{n}\left(\begin{array}{l}
n \\
k
\end{array}\right) x_{1}^{k} \Phi_{n-k}^{(\alpha, \ell)}\left(x_{2}, y, \nu\right) \\
& =\sum_{k=0}^{n}\left(\begin{array}{l}
n \\
k
\end{array}\right) x_{2}^{k} \Phi_{n-k}^{(\alpha, \ell)}\left(x_{1}, y, \nu\right) \quad\left(n \in \mathbb{N}_{0}, x_{1}, x_{2}, y \in \mathbb{C}\right) ; \\
& \Phi_{n}^{(\alpha, \ell)}\left(x, y_{1}+y_{2}, \nu\right)=\sum_{k=0}^{\left[\frac{n}{\ell}\right]} \frac{n ! y_{1}^{k}}{(n-\ell k) ! k !} \Phi_{n-\ell k}^{(\alpha, \ell)}\left(x, y_{2}, \nu\right) \\
& =\sum_{k=0}^{\left[\frac{n}{\ell}\right]} \frac{n ! y_{2}^{k}}{(n-\ell k) ! k !} \Phi_{n-\ell k}^{(\alpha, \ell)}\left(x, y_{1}, \nu\right) \\
& \left(n \in \mathbb{N}_{0}, x, y_{1}, y_{2} \in \mathbb{C}\right) \text {; } \\
& \Phi_{n}^{(\alpha, \ell)}(x, y, \nu)=\sum_{k=0}^{n}\left(\begin{array}{l}
n \\
k
\end{array}\right) x^{k} \Phi_{n-k}^{(\alpha, \ell)}(0, y, \nu) ; \quad\left(n \in \mathbb{N}_{0}, x, y \in \mathbb{C}\right) ; \\
& \Phi_{n}^{(\alpha, \ell)}(x, y, \nu)=\sum_{k=0}^{\left[\frac{n}{\ell}\right]} \frac{n ! y^{k}}{(n-\ell k) ! k !} \Phi_{n-\ell k}^{(\alpha, \ell)}(x, 0, \nu) \\
& \left(n \in \mathbb{N}_{0}, x, y \in \mathbb{C}\right) \text {; } \\
& \frac{\partial}{\partial x} \Phi_{n}^{(\alpha, \ell)}(x, y, \nu)=n \Phi_{n-1}^{(\alpha, \ell)}(x, y, \nu) \quad(n \in \mathbb{N}, x, y \in \mathbb{C}) ; \\
& \frac{\partial^{r}}{\partial x^{r}} \Phi_{n}^{(\alpha, \ell)}(x, y, \nu)=\frac{n !}{(n-r) !} \Phi_{n-r}^{(\alpha, \ell)}(x, y, \nu) \\
& (n, r \in \mathbb{N} \text { with } 1 \leq r \leq n ; x, y \in \mathbb{C}) \text {; } \\
& \frac{\partial}{\partial y} \Phi_{n}^{(\alpha, \ell)}(x, y, \nu)=\frac{n !}{(n-\ell) !} \Phi_{n-\ell}^{(\alpha, \ell)}(x, y, \nu) \\
& (n, \ell \in \mathbb{N} \text { with } 1 \leq \ell \leq n ; x, y \in \mathbb{C}) ; \\
& \int_{a}^{x} \Phi_{n}^{(\alpha, \ell)}(u, y, \nu) d u=\frac{\Phi_{n+1}^{(\alpha, \ell)}(x, y, \nu)-\Phi_{n+1}^{(\alpha, \ell)}(a, y, \nu)}{n+1} \\
& \left(n \in \mathbb{N}_{0}, a, x \in \mathbb{R}, y \in \mathbb{C}\right) .
\end{aligned}
$$




$$
\begin{aligned}
\int_{a}^{y} \Phi_{n}^{(\alpha, \ell)}(x, u, \nu) d u & =\frac{n !}{(n+\ell) !}\left\{\Phi_{n+\ell}^{(\alpha, \ell)}(x, y, \nu)-\Phi_{n+\ell}^{(\alpha, \ell)}(x, a, \nu)\right\} \\
(n & \left.\in \mathbb{N}_{0}, x \in \mathbb{C}, a, y \in \mathbb{R}\right) .
\end{aligned}
$$

Proof. From (1.23), we write

$$
\sum_{n=0}^{\infty} \Phi_{n}^{(\alpha, \ell)}\left(x_{1}+x_{2}, y, \nu\right) \frac{t^{n}}{n !}=\mathrm{e}^{x_{1} t} \cdot f(t, \alpha) \mathrm{e}^{x_{2} t+y t^{\ell}+h(t, \nu)} .
$$

Expanding $\mathrm{e}^{x_{1} t}$ as the Maclaurin series and using (1.23) to expand the second factor, with the aid of $(2.1)$ with $p=1$, we find

$$
\sum_{n=0}^{\infty} \Phi_{n}^{(\alpha, \ell)}\left(x_{1}+x_{2}, y, \nu\right) \frac{t^{n}}{n !}=\sum_{n=0}^{\infty} \sum_{k=0}^{n} \frac{x_{1}^{k}}{(n-k) ! k !} \Phi_{n-k}^{(\alpha, \ell)}\left(x_{2}, y, \nu\right) t^{n},
$$

which, upon equating the coefficients of $t^{n}$, yields the first equality of (2.4). For the second equality of (2.4), we just change the role of $x_{1}$ and $x_{2}$ in the above proof.

Similarly as in the proof of (2.4), with the aid of (2.1) with $p=\ell$, we prove (2.5).

Setting $x_{1}=x$ and $x_{2}=0$ in the first equality in (2.4), we obtain (2.6). Similarly, setting $y_{1}=y$ and $y_{2}=0$ in the first equality in (2.5), we get (2.7).

Differentiating both sides of (2.6) with respect to the variable $x$, we have

$$
\begin{aligned}
\frac{\partial}{\partial x} \Phi_{n}^{(\alpha, \ell)}(x, y, \nu) & =\sum_{k=1}^{n} k\left(\begin{array}{l}
n \\
k
\end{array}\right) x^{k-1} \Phi_{n-k}^{(\alpha, \ell)}(0, y, \nu) \\
& =n \sum_{k=0}^{n-1}\left(\begin{array}{c}
n-1 \\
k
\end{array}\right) x^{k} \Phi_{n-1-k}^{(\alpha, \ell)}(0, y, \nu) \\
& =n \Phi_{n-1}^{(\alpha, \ell)}(x, y, \nu)
\end{aligned}
$$

where the identity (2.6) is used for the last equality. This proves (2.8).

Then, differentiating both sides of (2.8) with respect to the variable $x$ by using the identity (2.8) on the right side of each resulting identity, consecutively, $r-1$ times, we obtain (2.9).

Differentiating both sides of (2.7) with respect to the variable $y$, we have

$$
\frac{\partial}{\partial y} \Phi_{n}^{(\alpha, \ell)}(x, y, \nu)=\sum_{k=1}^{\left[\frac{n}{\ell}\right]} \frac{n ! y^{k-1}}{(n-\ell k) !(k-1) !} \Phi_{n-\ell k}^{(\alpha, \ell)}(x, 0, \nu) .
$$

Taking $k-1=k^{\prime}$ on the right side of (2.14) and considering

we get

$$
\left[\frac{n}{\ell}\right]-1=\left[\frac{n}{\ell}-1\right]=\left[\frac{n-\ell}{\ell}\right]
$$

$$
\frac{\partial}{\partial y} \Phi_{n}^{(\alpha, \ell)}(x, y, \nu)=\frac{n !}{(n-\ell) !} \sum_{k=0}^{\left[\frac{n-\ell}{\ell}\right]} \frac{(n-\ell) ! y^{k}}{(n-\ell-\ell k) ! k !} \Phi_{n-\ell-\ell k}^{(\alpha, \ell)}(x, 0, \nu),
$$

which, upon using (2.7), proves (2.10).

Replacing $x$ by $u$ in (2.8) and integrating both sides of the resulting identity with respect to the variable $u$ from $a$ to $x$ by using the fundamental theorem of calculus, and substituting $n+1$ for $n$ in the last resulting identity, we obtain (2.11).

Similarly as in getting (2.11), using (2.10), we get (2.12). 


\section{Power sum-Laguerre-Hermite polynomials}

Here, replacing $x$ by $y$ and $\nu$ by $z$ in (1.9) and setting $h(t, z)=z t^{2}$ and

$$
f(x ; t, \mathrm{n})=\frac{\mathrm{e}^{(\mathrm{n}+1) t}-1}{\mathrm{e}^{t}-1} C_{0}(x t),
$$

we introduce a new class of power sum-Laguerre-Hermite polynomials ${ }_{H}^{S} L_{n}(x, y, z ; \mathrm{n})$ by the following generating function:

$$
\frac{\mathrm{e}^{(\mathrm{n}+1) t}-1}{\mathrm{e}^{t}-1} \mathrm{e}^{y t+z t^{2}} C_{0}(x t)=\sum_{n=0}^{\infty}{ }_{H}^{S} L_{n}(x, y, z ; \mathrm{n}) \frac{t^{n}}{n !} \quad(|t|<2 \pi) .
$$

Now, we present various implicit summation formulae for the power sum-LaguerreHermite polynomials.

Theorem 3.1. The following implicit summation formulas for the power sum-LaguerreHermite polynomials hold.

$$
\begin{gathered}
{ }_{H}^{S} L_{n}(x, y, 0 ; \mathrm{n})=\sum_{k=0}^{n}\left(\begin{array}{l}
n \\
k
\end{array}\right) L_{n-k}(x, y) S_{k}(\mathrm{n}) \quad\left(n \in \mathbb{N}_{0} ; \mathrm{n} \in \mathbb{N}\right) ; \\
{ }_{H}^{S} L_{n}(x, y, z ; \mathrm{n})=n ! \sum_{r=0}^{n} \sum_{k=0}^{n-r} \frac{(-1)^{r} x^{r} H_{n-k-r}(y, z) S_{k}(\mathrm{n})}{(r !)^{2} k !(n-k-r) !} \quad\left(n \in \mathbb{N}_{0} ; \mathrm{n} \in \mathbb{N}\right) ; \\
{ }_{H}^{S} L_{n}(x, u+v, z ; \mathrm{n})=\sum_{k=0}^{n}\left(\begin{array}{l}
n \\
k
\end{array}\right) u^{k}{ }_{H}^{S} L_{n-k}(x, v, z ; \mathrm{n}) \quad\left(n \in \mathbb{N}_{0} ; \mathrm{n} \in \mathbb{N}\right) ; \\
{ }_{H}^{S} L_{n}(x, y, a+b ; \mathrm{n})=\sum_{k=0}^{\left[\frac{n}{2}\right]} \frac{n !}{k !(n-2 k) !} b_{H}^{k} L_{n-2 k}(x, y, a ; \mathrm{n}) \quad\left(n \in \mathbb{N}_{0} ; \mathrm{n} \in \mathbb{N}\right) .
\end{gathered}
$$

Proof. Setting $z=0$ in (3.1) and using (1.2) and (1.16) with the aid of (2.1) with $p=1$, we obtain

$$
\sum_{n=0}^{\infty}{ }_{H}^{S} L_{n}(x, y, z ; \mathrm{n}) \frac{t^{n}}{n !}=\sum_{n=0}^{\infty} \sum_{k=0}^{n} L_{n-k}(x, y) S_{k}(\mathrm{n}) \frac{t^{n}}{(n-k) ! k !},
$$

which, upon equating the coefficients of $t^{n}$, yields the desired result (3.2).

The other identities can be proved as in the proof of (3.2). We omit the details.

\section{Generalized Laguerre-Euler polynomials}

Here, replacing $x$ by $y$ and $\nu$ by $z$ in $(1.9)$ and $f(x ; t, \alpha)=\left(\frac{2}{a^{t}+b^{t}}\right)^{\alpha} C_{0}(x t)$, we introduce a new class of the generalized Laguerre-Euler polynomials.

Let $\alpha \in \mathbb{R}$ or $\mathbb{C}$ be a parameter. Also, let $a, b \in \mathbb{R}^{+}$with $a \neq b$. The generalized Euler polynomials $E_{n}^{(\alpha)}(x, y, z ; a, b, \mathrm{e})$ are defined by the following generating function

$$
\begin{gathered}
\left(\frac{2}{a^{t}+b^{t}}\right)^{\alpha} \mathrm{e}^{y t+h(t, z)} C_{0}(x t)=\sum_{n=0}^{\infty} E_{n}^{(\alpha)}(x, y, z ; a, b, \mathrm{e}) \frac{t^{n}}{n !} \\
\left(x \in \mathbb{R} ;|t|<\frac{2 \pi}{|\ln a-\ln b|}\right) .
\end{gathered}
$$

In particular, setting $h(t, z)=z t^{2}$ in (4.1), we get 
Let $\alpha \in \mathbb{R}$ or $\mathbb{C}$ be a parameter. Also, let $a, b \in \mathbb{R}^{+}$with $a \neq b$. The generalized Laguerre-Euler polynomials ${ }_{L} E_{n}^{(\alpha)}(x, y, z ; a, b, \mathrm{e})$ are defined by

We have

$$
\begin{gathered}
\left(\frac{2}{a^{t}+b^{t}}\right)^{\alpha} \mathrm{e}^{y t+z t^{2}} C_{0}(x t)=\sum_{n=0}^{\infty}{ }_{L} E_{n}^{(\alpha)}(x, y, z ; a, b, \mathrm{e}) \frac{t^{n}}{n !} \\
\left(x \in \mathbb{R} ;|t|<\frac{2 \pi}{|\ln a-\ln b|}\right) .
\end{gathered}
$$

$$
{ }_{L} E_{n}^{(\alpha)}(x, y, z ; a, b, \mathrm{e})=\sum_{m=0}^{n} \sum_{k=0}^{\left[\frac{m}{2}\right]} \frac{E_{n-m}^{(\alpha)} L_{m-2 k}(x, y) z^{k} n !}{(m-2 k) ! k !(n-m) !} .
$$

Remark 4.1. Consider some special cases of (4.2).

(i) The case $x=0$ of (4.2) reduces to the known generalized Hermite-Bernoulli polynomials defined by (see [24])

$$
\begin{gathered}
\left(\frac{2}{a^{t}+b^{t}}\right)^{\alpha} \quad \mathrm{e}^{y t+z t^{2}}=\sum_{n=0}^{\infty}{ }_{H} E_{n}^{(\alpha)}(y, z ; a, b, \mathrm{e}) \frac{t^{n}}{n !} \\
\left(|t|<\frac{2 \pi}{|\ln a-\ln b|}\right) .
\end{gathered}
$$

(ii) The case $x=z=0$ of (4.2) reduces to the known generalized Euler polynomials defined by (see $[20]$ )

$$
\begin{gathered}
\left(\frac{2}{a^{t}+b^{t}}\right)^{\alpha} \mathrm{e}^{y t}=\sum_{n=0}^{\infty} E_{n}^{(\alpha)}(y ; a, b, \mathrm{e}) \frac{t^{n}}{n !} \\
\left(|t|<\frac{2 \pi}{|\ln a-\ln b|}\right) .
\end{gathered}
$$

(iii) The case $x=y=z=0$ of (4.2) reduces to the generalized Euler number $E_{n}^{(\alpha)}(a, b)$ defined by

$$
\begin{gathered}
\left(\frac{2}{a^{t}+b^{t}}\right)^{\alpha}=\sum_{n=0}^{\infty} E_{n}^{(\alpha)}(a, b) \frac{t^{n}}{n !} \\
\left(|t|<\frac{2 \pi}{|\ln a-\ln b|}\right) .
\end{gathered}
$$

We find that $E_{n}^{(1)}(a, b)=E_{n}(a, b)$ in $(1.17)$ and

$$
E_{n}^{(\alpha+\beta)}(a, b)=\sum_{k=0}^{n}\left(\begin{array}{l}
n \\
k
\end{array}\right) E_{k}^{(\alpha)}(a, b) E_{n-k}^{(\beta)}(a, b) \quad\left(n \in \mathbb{N}_{0}\right) .
$$

Here, we present various implicit summation formulae for the generalized Laguerre-Euler polynomials.

Theorem 4.2. Let $\alpha, \beta \in \mathbb{R}$ or $\mathbb{C}$ be parameters. Also, let $a, b \in \mathbb{R}^{+}$with $a \neq b$. Further, let $u, v, w, x, y, z \in \mathbb{R}$, and $n \in \mathbb{N}_{0}$. Then the following implicit summation formulas for the generalized Laguerre-Euler polynomials in (4.2) hold:

$$
\begin{aligned}
& { }_{L} E_{m+n}^{(\alpha)}(x, w, z ; a, b, \mathrm{e}) \\
& \quad=\sum_{s=0}^{m} \sum_{k=0}^{n}\left(\begin{array}{c}
m \\
s
\end{array}\right)\left(\begin{array}{l}
n \\
k
\end{array}\right)(w-y)^{s+k}{ }_{L} E_{m+n-s-k}^{(\alpha)}(x, y, z ; a, b, \mathrm{e}) ;
\end{aligned}
$$




$$
\begin{gathered}
{ }_{L} E_{n}^{(\alpha)}(x, y+\alpha, z ; a, b, \mathrm{e})=n ! \sum_{j=0}^{\left[\frac{n}{2}\right]} \sum_{k=0}^{n-2 j} \frac{(-1)^{k} x^{k} z^{j} E_{n-2 j-k}^{(\alpha)}\left(y ; \frac{a}{\mathrm{e}}, \frac{b}{\mathrm{e}}, \mathrm{e}\right)}{(n-2 j-k) ! j !(k !)^{2}} \\
{ }_{L} E_{n}^{(\alpha+\beta)}(x, y+v, z ; a, b, \mathrm{e}) \\
=\sum_{k=0}^{n}\left(\begin{array}{l}
n \\
k
\end{array}\right){ }_{L} E_{n-k}^{(\alpha)}(x, y, z ; a, b, \mathrm{e}) E_{k}^{(\beta)}(v ; a, b, \mathrm{e}) ; \\
{ }_{L} E_{n}^{(\alpha+\beta)}(x, y+z, v+u ; a, b, \mathrm{e}) \\
=\sum_{k=0}^{n}\left(\begin{array}{l}
n \\
k
\end{array}\right) E_{n-k}^{(\alpha)}(x, z, v ; a, b, \mathrm{e})_{H} E_{k}^{(\beta)}(y, u ; a, b, \mathrm{e}) ; \\
{ }_{L} E_{n}^{(\alpha)}(x, y, z ; a, b, \mathrm{e})=n ! \sum_{j=0}^{\left[\frac{n}{2}\right]} \sum_{k=0}^{n-2 j} \frac{E_{k}^{(\alpha)}(a, b, \mathrm{e}) L_{n-k-2 j}(x, y) z^{j}}{k ! j !(n-k-2 j) !} .
\end{gathered}
$$

Proof. For (4.8), replacing $t$ by $t+u$ in (4.2) and using the binomial theorem, we have

$$
\begin{aligned}
& \left(\frac{2}{a^{t+u}+b^{t+u}}\right)^{\alpha} \mathrm{e}^{y(t+u)+z(t+u)^{2}} C_{0}(x(t+u)) \\
& =\sum_{n=0}^{\infty}{ }_{L} E_{n}^{(\alpha)}(x, y, z ; a, b, \mathrm{e}) \frac{(t+u)^{n}}{n !} \\
& =\sum_{n=0}^{\infty} \sum_{m=0}^{n}{ }_{L} E_{n}^{(\alpha)}(x, y, z ; a, b, \mathrm{e}) \frac{t^{n-m} u^{m}}{(n-m) ! m !} .
\end{aligned}
$$

Using (2.2) with $p=1$ in the last double summation in (4.13), we obtain

$$
\begin{aligned}
& \left(\frac{2}{a^{t+u}+b^{t+u}}\right)^{\alpha} \mathrm{e}^{z(t+u)^{2}} C_{0}(x(t+u)) \\
& =\mathrm{e}^{-y(t+u)} \sum_{n=0}^{\infty} \sum_{m=0}^{\infty}{ }_{L} E_{n+m}^{(\alpha)}(x, y, z ; a, b, \mathrm{e}) \frac{t^{n} u^{m}}{n ! m !} .
\end{aligned}
$$

Since the left side of (4.14) is independent of the variable $y$, we introduce another variable $w$ for the variable $y$ in the right side of (4.14) and equate the two resulting identities to find

$$
\begin{aligned}
& \sum_{n=0}^{\infty} \sum_{m=0}^{\infty}{ }_{L} E_{n+m}^{(\alpha)}(x, w, z ; a, b, \mathrm{e}) \frac{t^{n} u^{m}}{n ! m !} \\
& =\mathrm{e}^{(w-y)(t+u)} \sum_{n=0}^{\infty} \sum_{m=0}^{\infty}{ }_{L} E_{n+m}^{(\alpha)}(x, y, z ; a, b, \mathrm{e}) \frac{t^{n} u^{m}}{n ! m !} .
\end{aligned}
$$

We use (2.3) to find

$$
\mathrm{e}^{(w-y)(t+u)}=\sum_{N=0}^{\infty}(w-y)^{N} \frac{(t+u)^{N}}{N !}=\sum_{k, s=0}^{\infty}(w-y)^{k+s} \frac{t^{k} u^{s}}{k ! s !} .
$$

Using (4.16) in the right side of (4.15) and applying (2.1) with $p=1$ in the resulting quadruple series, two times, we get

$$
\begin{aligned}
& \sum_{n=0}^{\infty} \sum_{m=0}^{\infty}{ }_{L} E_{n+m}^{(\alpha)}(x, w, z ; a, b, \mathrm{e}) \frac{t^{n} u^{m}}{n ! m !} \\
& =\sum_{n=0}^{\infty} \sum_{m=0}^{\infty} \sum_{k=0}^{n} \sum_{s=0}^{m}{ }_{L} E_{n+m-s-k}^{(\alpha)}(x, y, z ; a, b, \mathrm{e})(w-y)^{k+s} \frac{t^{n} u^{m}}{(n-k) ! k !(m-s) ! s !} .
\end{aligned}
$$


Finally, equating the coefficients of $t^{n}$ and $u^{m}$ in both sides of (4.17), consecutively, we obtain the identity (4.8).

For (4.9), we find from (4.2) that

$$
\sum_{n=0}^{\infty}{ }_{L} E_{n}^{(\alpha)}(x, y+\alpha, z ; a, b, \mathrm{e}) \frac{t^{n}}{n !}=\left(\frac{2}{\left(\frac{a}{\mathrm{e}}\right)^{t}+\left(\frac{b}{\mathrm{e}}\right)^{t}}\right)^{\alpha} \mathrm{e}^{y t} \cdot \mathrm{e}^{z t^{2}} \cdot C_{0}(x t)
$$

By using (4.5) and (2.1) with $p=2$, we have

$$
\begin{aligned}
\left(\frac{2}{\left(\frac{a}{\mathrm{e}}\right)^{t}+\left(\frac{b}{\mathrm{e}}\right)^{t}}\right)^{\alpha} \mathrm{e}^{y t} \cdot \mathrm{e}^{z t^{2}} & =\sum_{n=0}^{\infty} E_{n}^{(\alpha)}\left(y ; \frac{a}{\mathrm{e}}, \frac{b}{\mathrm{e}}, \mathrm{e}\right) \frac{t^{n}}{n !} \cdot \sum_{j=0}^{\infty} \frac{z^{j} t^{2 j}}{j !} \\
& =\sum_{n=0}^{\infty} \sum_{j=0}^{\left[\frac{n}{2}\right]} E_{n-2 j}^{(\alpha)}\left(y ; \frac{a}{\mathrm{e}}, \frac{b}{\mathrm{e}}, \mathrm{e}\right) z^{j} \frac{t^{n}}{(n-2 j) ! j !} .
\end{aligned}
$$

Setting the result (4.19) in (4.18) and using (1.4) with $n=0$, with the help of (2.1) with $p=1$, we obtain

$$
\begin{aligned}
& \sum_{n=0}^{\infty}{ }_{L} E_{n}^{(\alpha)}(x, y+\alpha, z ; a, b, \mathrm{e}) \frac{t^{n}}{n !} \\
& \quad=\sum_{n=0}^{\infty}\left\{\sum_{j=0}^{\left[\frac{n}{2}\right]} \sum_{k=0}^{n-2 j} E_{n-2 j-k}^{(\alpha)}\left(y ; \frac{a}{\mathrm{e}}, \frac{b}{\mathrm{e}}, \mathrm{e}\right) \frac{z^{j} x^{k}(-1)^{k}}{(n-2 j-k) ! j !(k !)^{2}}\right\} t^{n} .
\end{aligned}
$$

Finally, equating the coefficients of $t^{n}$ on both sides of (4.20), we get the identity (4.9).

Similarly as above, we can prove the other identities. We omit the details.

\section{Symmetry identities for the generalized Laguerre-Euler polynomials}

A number of interesting symmetry identities for various polynomials have been presented (see, e.g., [12-18, 29,30]). Here, we give symmetry identities for the generalized Laguerre-Euler polynomials ${ }_{L} E_{n}^{(\alpha)}(x, y, z ; a, b, \mathrm{e})$ in (4.2). To do this, we consider the following function:

$$
\begin{aligned}
g(t):= & \left\{\frac{4}{\left(c^{a t}+d^{a t}\right)\left(c^{b t}+d^{b t}\right)}\right\}^{\alpha}\left\{\frac{4}{\left(c^{a t}+d^{a t}\right)\left(c^{b t}+d^{b t}\right)}\right\}^{\beta} \\
& \times \mathrm{e}^{(a+b)\left(y_{1}+y_{2}\right) t+\left(a^{2}+b^{2}\right)\left(z_{1}+z_{2}\right) t^{2}} \\
& \times C_{0}\left(x_{1} a t\right) C_{0}\left(x_{1} b t\right) C_{0}\left(x_{2} a t\right) C_{0}\left(x_{2} b t\right) .
\end{aligned}
$$

We see that the function $g(t)$ in (5.1) is symmetric with respect to $\alpha$ and $\beta, a$ and $b, c$ and $d, x_{1}$ and $x_{2}, y_{1}$ and $y_{2}, z_{1}$ and $z_{2}$, respectively. So, to make the generalized Laguerre-Euler polynomials in (4.2), we have 16 combinations. Then we will get 15 symmetry identities for the generalized Laguerre-Euler polynomials in (4.2), two of which will be asserted in the following theorem and the other 13 of which are left to the interested reader.

Theorem 5.1. Let $\alpha, \beta \in \mathbb{R}$ or $\mathbb{C}$ be parameters. Also, let $c, d \in \mathbb{R}^{+}$with $c \neq d$. Further, let $a, b, x_{1}, x_{2}, y_{1}, y_{2}, z_{1}, z_{2} \in \mathbb{R}$ and $n \in \mathbb{N}_{0}$. Then

$$
\begin{aligned}
& \sum_{r=0}^{n} \sum_{m=0}^{n-r} \sum_{s=0}^{r}{ }_{L} E_{n-m-r}^{(\alpha)}\left(x_{1}, y_{1}, z_{1} ; c, d, \mathrm{e}\right){ }_{L} E_{m}^{(\alpha)}\left(x_{1}, y_{1}, z_{1} ; c, d, \mathrm{e}\right) \\
& \times{ }_{L} E_{r-s}^{(\beta)}\left(x_{2}, y_{2}, z_{2} ; c, d, \mathrm{e}\right){ }_{L} E_{s}^{(\beta)}\left(x_{2}, y_{2}, z_{2} ; c, d, \mathrm{e}\right) \frac{a^{n-m-s} b^{m+s}}{(n-m-r) ! m !(r-s) ! s !}
\end{aligned}
$$




$$
\begin{aligned}
& =\sum_{r=0}^{n} \sum_{m=0}^{n-r} \sum_{s=0}^{r}{ }_{L} E_{n-m-r}^{(\alpha)}\left(x_{2}, y_{2}, z_{2} ; c, d, \mathrm{e}\right){ }_{L} E_{m}^{(\alpha)}\left(x_{2}, y_{2}, z_{2} ; c, d, \mathrm{e}\right) \\
& \times{ }_{L} E_{r-s}^{(\beta)}\left(x_{1}, y_{1}, z_{1} ; c, d, \mathrm{e}\right){ }_{L} E_{s}^{(\beta)}\left(x_{1}, y_{1}, z_{1} ; c, d, \mathrm{e}\right) \frac{a^{n-m-s} b^{m+s}}{(n-m-r) ! m !(r-s) ! s !} \\
& =\sum_{r=0}^{n} \sum_{m=0}^{n-r} \sum_{s=0}^{r} E_{n-m-r}^{(\beta)}\left(x_{2}, y_{1}, z_{1} ; c, d, \mathrm{e}\right){ }_{L} E_{m}^{(\beta)}\left(x_{2}, y_{1}, z_{1} ; c, d, \mathrm{e}\right) \\
& \times{ }_{L} E_{r-s}^{(\alpha)}\left(x_{1}, y_{2}, z_{2} ; c, d, \mathrm{e}\right){ }_{L} E_{s}^{(\alpha)}\left(x_{1}, y_{2}, z_{2} ; c, d, \mathrm{e}\right) \frac{b^{n-m-s} a^{m+s}}{(n-m-r) ! m !(r-s) ! s !} .
\end{aligned}
$$

Proof. We try to combine $g(t)$ as follows:

$$
\begin{aligned}
g(t) & =\left\{\frac{2}{c^{a t}+d^{a t}}\right\}^{\alpha} \mathrm{e}^{a y_{1} t+a^{2} z_{1} t} C_{0}\left(x_{1} a t\right) \\
& \times\left\{\frac{2}{c^{b t}+d^{b t}}\right\}^{\alpha} \mathrm{e}^{b y_{1} t+b^{2} z_{1} t} C_{0}\left(x_{1} b t\right) \\
& \times\left\{\frac{2}{c^{a t}+d^{a t}}\right\}^{\beta} \mathrm{e}^{a y_{2} t+a^{2} z_{2} t} C_{0}\left(x_{2} a t\right) \\
& \times\left\{\frac{2}{c^{b t}+d^{b t}}\right\}^{\beta} \mathrm{e}^{b y_{2} t+b^{2} z_{2} t} C_{0}\left(x_{2} b t\right),
\end{aligned}
$$

which, upon using (4.2), gives

$$
\begin{aligned}
g(t) & =\sum_{n=0}^{\infty}{ }_{L} E_{n}^{(\alpha)}\left(x_{1}, y_{1}, z_{1} ; c, d, \mathrm{e}\right) \frac{(a t)^{n}}{n !} \\
& \times \sum_{m=0}^{\infty}{ }_{L} E_{m}^{(\alpha)}\left(x_{1}, y_{1}, z_{1} ; c, d, \mathrm{e}\right) \frac{(b t)^{m}}{m !} \\
& \times \sum_{r=0}^{\infty}{ }_{L} E_{r}^{(\beta)}\left(x_{2}, y_{2}, z_{2} ; c, d, \mathrm{e}\right) \frac{(a t)^{r}}{r !} \\
& \times \sum_{s=0}^{\infty}{ }_{L} E_{s}^{(\beta)}\left(x_{2}, y_{2}, z_{2} ; c, d, \mathrm{e}\right) \frac{(b t)^{s}}{s !}
\end{aligned}
$$

Now, we apply (2.1) with $p=1$ to combine the first and second series into a single series and the third and fourth series into another single series. Then we use (2.1) with $p=1$ to combine the two resulting single series into one series to find

$$
\begin{aligned}
& g(t)=\sum_{n=0}^{\infty}\left\{\sum_{r=0}^{n} \sum_{m=0}^{n-r} \sum_{s=0}^{r}{ }_{L} E_{n-m-r}^{(\alpha)}\left(x_{1}, y_{1}, z_{1} ; c, d, \mathrm{e}\right){ }_{L} E_{m}^{(\alpha)}\left(x_{1}, y_{1}, z_{1} ; c, d, \mathrm{e}\right)\right. \\
& \left.\times{ }_{L} E_{r-s}^{(\beta)}\left(x_{2}, y_{2}, z_{2} ; c, d, \mathrm{e}\right){ }_{L} E_{s}^{(\beta)}\left(x_{2}, y_{2}, z_{2} ; c, d, \mathrm{e}\right) \frac{a^{n-m-s} b^{m+s}}{(n-m-r) ! m !(r-s) ! s !}\right\} t^{n} .
\end{aligned}
$$

Considering another combination of $g(t)$ as in (5.4), similarly as above, we can get another single series of $g(t)$ as in (5.6). Then, equating the coefficients of $t^{n}$ in both sides of the two single series, we can find 15 identities, two of which are recorded. 


\section{Concluding remarks}

The results presented here, being very general, can be specialised to yield a number of known and new identities involving relatively simple and familiar polynomials. For example, setting $x=0$ in (4.8), we have

$$
\begin{aligned}
& { }_{H} E_{m+n}^{(\alpha)}(w, z ; a, b, \mathrm{e}) \\
& \quad=\sum_{s=0}^{m} \sum_{k=0}^{n}\left(\begin{array}{c}
m \\
s
\end{array}\right)\left(\begin{array}{l}
n \\
k
\end{array}\right)(w-y)^{s+k}{ }_{H} E_{m+n-s-k}^{(\alpha)}(y, z ; a, b, \mathrm{e}) .
\end{aligned}
$$

The power sum-Laguerre-Hermite polynomials ${ }_{H}^{S} L_{n}(x, y, z ; \mathrm{n})$ in (3) and the generalized Laguerre-Euler polynomials $E_{n}^{(\alpha)}(x, y, z ; a, b, \mathrm{e})$ in $(4.2)$ can be further extended and have their differential and integral formulas as in Theorem 2.2.

Acknowledgment. The authors would like to express their deep thanks for the reviewer whose useful comments improve this paper as it stands.

\section{References}

[1] L.C. Andrews, Special Functions for Engineer and Mathematician, Macmillan Company, New York, 1985.

[2] E.T. Bell, Exponential polynomials, Ann. Math. 35 (2), 258-277, 1934.

[3] G. Betti and P.E. Ricci, Multidimensional extensions of the Bernoulli and Appell polynomials, Taiwanese J. Math. 8 (3), 415-428, 2004.

[4] J. Choi, Notes on formal manipulations of double series, Commun. Korean Math. Soc. 18 (4), 781-789, 2003.

[5] J. Choi, N.U. Khan and T. Usman, A note on Legendre-based multi poly-Euler polynomials, Bull. Iran. Math. Soc. 44, 707-717, 2018.

[6] G. Dattoli, S. Lorenzutta and C. Cesarano, Finite sums and generalized forms of Bernoulli polynomials, Rend. Mat. 19, 385-391, 1999.

[7] G. Dattoli and A. Torre, Theory and Applications of Generalized Bessel Function, Aracne, Rome, 1996.

[8] G. Dattoli and A. Torre, Operational methods and two variable Laguerre polynomials, Atti Acad. Torino 132, 1-7, 1998.

[9] G. Dattoli, A. Torre and A.M. Mancho, The generalized Laguerre polynomials, the associated Bessel functions and applications to propagation problems, Radiat. Phys. Chem. 59, 229-237, 2000.

[10] R. Dere and Y. Simsek, Hermite base Bernoulli type polynomials on the umbral algebra, Russian J. Math. Phys. 22 (1), 1-5, 2015.

[11] B.N. Guo and F. Qi, Generalization of Bernoulli polynomials, J. Math. Ed. Sci. Tech. 33 (3), 428-431, 2002.

[12] N.U. Khan and T. Usman, A new class of Laguerre-based generalized Apostol polynomials, Fasciculli. Math. 57, 67-89, 2016.

[13] N.U. Khan and T. Usman, A new class of Laguerre-based poly-Euler and multi polyEuler polynomials, J. Anal. Num. Theor. 4 (2), 113-120, 2016.

[14] N.U. Khan and T. Usman, A new class of Laguerre poly-Bernoulli numbers and polynomials, Adv. Stud. Contemporary Math. 27 (2), 229-241, 2017.

[15] N.U. Khan, T. Usman and A. Aman, Generating functions for Legendre-Based polyBernoulli numbers and polynomials, Honam Math. J. 39 (2), 217-231, 2017.

[16] N.U. Khan, T. Usman and J. Choi, Certain generating function of Hermite-BernoulliLaguerre polynomials, Far East J. Math. Sci. 101 (4), 893-908, 2017. 
[17] N.U. Khan, T. Usman and J. Choi, A new generalization of Apostol type LaguerreGenocchi polynomials, C. R. Acad. Sci. Paris, Ser. I, 355, 607-617, 2017.

[18] N.U. Khan, T. Usman and J. Choi, A new class of generalized polynomials associated with Laguerre and Bernoulli polynomials, Turkish J. Math. 43, 486-497, 2019.

[19] B. Kurt and Y. Simsek, Notes on generalization of the Bernoulli type polynomials, Appl. Math. Comput. 218, 906-911, 2011.

[20] Q.-M. Luo, B.N. Guo, F. Qi and L. Debnath, Generalization of Bernoulli numbers and polynomials, Int. J. Math. Math. Sci. 59, 3769-3776, 2003.

[21] Q.-M. Luo, F. Qi and L. Debnath, Generalization of Euler numbers and polynomials, Int. J. Math. Math. Sci. 61, 3893-3901, 2003.

[22] L.M. Milne-Thomsons, Two classes of generalized polynomials, Proc. London Math. Soc. 35 (1), 514-522, 1933.

[23] M.A. Pathan, A new class of generalized Hermite-Bernoulli polynomials, Georgian Math. J. 19, 559-573, 2012.

[24] M.A. Pathan and W.A. Khan, A new class of generalized polynomials associated with Hermite and Euler polynomials, Mediterr. J. Math. 13 (3), 913-928, 2016.

[25] F. Qi and B.N. Guo, Generalization of Bernoulli polynomials, RGMIA Res. Rep. Coll. 4 (4), Article 10, 691-695, 2001.

[26] E.D. Rainville, Special Functions, Macmillan Company, New York, 1960; Reprinted by Chelsea Publishing Company, Bronx, New York, 1971.

[27] H.M. Srivastava and J. Choi, Zeta and q-Zeta Functions and Associated Series and Integrals, Elsevier Science Publishers, Amsterdam, London and New York, 2012.

[28] H.M. Srivastava and H.L. Manocha, A Treatise on Generating Functions, Halsted Press (Ellis Horwood Limited, Chichester), John Wiley and Sons, New York, Chichester, Brisbane and Toronto, 1984.

[29] S. Yang, An identity of symmetry for the Bernoulli polynomials, Discrete Math. 308, 550-554, 2008.

[30] Z. Zhang and H. Yang, Several identities for the generalized Apostol Bernoulli polynomials, Comput. Math. Appl. 56 (12), 2993-2999, 2008. 